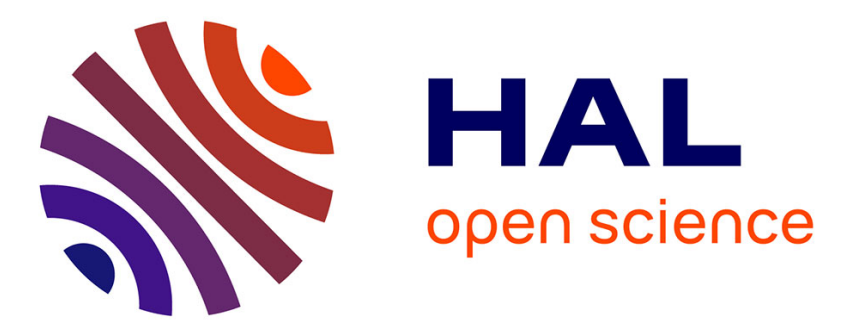

\title{
Mesure de durées de vie au moyen de champs hyperfréquences
}

\author{
J. Gerber, J.P. Vivien, A. Macher, R. Armbruster
}

\section{To cite this version:}

J. Gerber, J.P. Vivien, A. Macher, R. Armbruster. Mesure de durées de vie au moyen de champs hyperfréquences. Revue de Physique Appliquée, 1969, 4 (2), pp.218-220. 10.1051/rphysap:0196900402021800 . jpa-00243228

\section{HAL Id: jpa-00243228 https://hal.science/jpa-00243228}

Submitted on 1 Jan 1969

HAL is a multi-disciplinary open access archive for the deposit and dissemination of scientific research documents, whether they are published or not. The documents may come from teaching and research institutions in France or abroad, or from public or private research centers.
L'archive ouverte pluridisciplinaire HAL, est destinée au dépôt et à la diffusion de documents scientifiques de niveau recherche, publiés ou non, émanant des établissements d'enseignement et de recherche français ou étrangers, des laboratoires publics ou privés. 


\title{
MESURE DE DURÉES DE VIE AU MOYEN DE GHAMPS HYPERFRÉQUENGES
}

\author{
J. GERBER, J. P. VIVIEN, A. MACHER et R. ARMBRUSTER, \\ Institut de Recherches Nucléaires, Strasbourg-Cronenbourg.
}

\begin{abstract}
Résumé. - Des champs électriques synchrones pulsent le faisceau, puis modulent en énergie des électrons $K$, ou bien modulent les deux électrons $K$ d'une cascade.

Abstract. - Two methods of measuring lifetimes using UHF electric fields are presented. A simulation method gives directly the shape of the decay spectrum.
\end{abstract}

Dans l'étude des noyaux déformés impairs, il est d'un grand intérêt de déterminer les durées de vie de niveaux appartenant à la même bande rotationnelle. Si les premiers niveaux ont été mesurés, les deuxièmes niveaux ne le sont pas et l'excitation coulombienne de particules ${ }^{4} \mathrm{He}$ permet de les peupler correctement avec un bruit de fond réduit. Les transitions observées sont de faible énergie et en général bien converties. Une pulsation du faisceau synchrone avec une modulation en énergie des électrons de conversion $\mathrm{K}$ de ces transitions permet de mesurer les durées de vie avec une bonne précision dans la gamme de $10^{-10}$ à $10^{-11} \mathrm{~s}$. Dans le cás des sources radioactives, nous avons mis au point un système de double modulation qu'il est possible d'étendre aux réactions nucléaires dans le cas où celles-ci conduisent à une ambiguïté sur les populations des niveaux.

Deux méthodes, semblables dans le principe, permettent de déclencher les transitions considérées, d'une part en peuplant directement le niveau par des paquets de particules espacés de 200 ps, d'autre part en modulant en énergie l'électron $\mathrm{K}$ de la première transition de la cascade par des champs électriques de période 400 ps. Dans ces deux cas de population, l'électron $\mathrm{K}$ de la transition à mesurer est modulé en énergie par un champ électrique synchrone (fig. 1 et 2 ).

La similitude de ces deux méthodes réside dans les distributions obtenues : pour l'une dans la distribution spatiale du faisceau défléchi par un champ électrique sinusoïdal transversal, pour l'autre dans la distribution du spectre d'énergie de la raie d'électrons $K$ modulée par un champ électrique sinusoïdal parallèle à leur trajectoire. Dans les deux cas, le bruit est supposé de type gaussien.

Pour l'exploitation des spectres obtenus dans les deux méthodes, il faut calculer les distributions plus complexes quand elles contiennent l'information de durée de vie. Les facteurs essentiels qui interviennent sont : i) la différence de phase entre les deux champs synchrones qui, à l'encontre des variations relatives de


FIG. 1. - Schéma expérimental (1re méthode) : utilisation d'un faisceau pulsé ; les électrons de conversion $\mathrm{K}$ traversent la cavité modulatrice et sont détectés après passage dans le spectromètre.

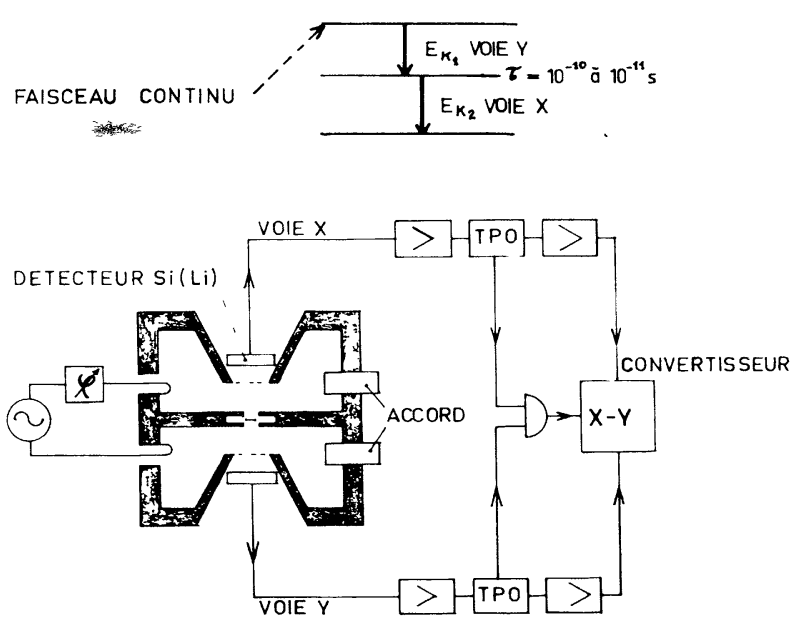

FIG. 2. - Schéma expérimental ( $2^{\mathrm{e}}$ méthode) : utilisation d'une source; les électrons de conversion $\mathrm{K}_{1}$ et $\mathrm{K}_{2}$ traversent le double convertisseur temps-énergie constitué par une double cavité hyperfréquence modulatrice. 

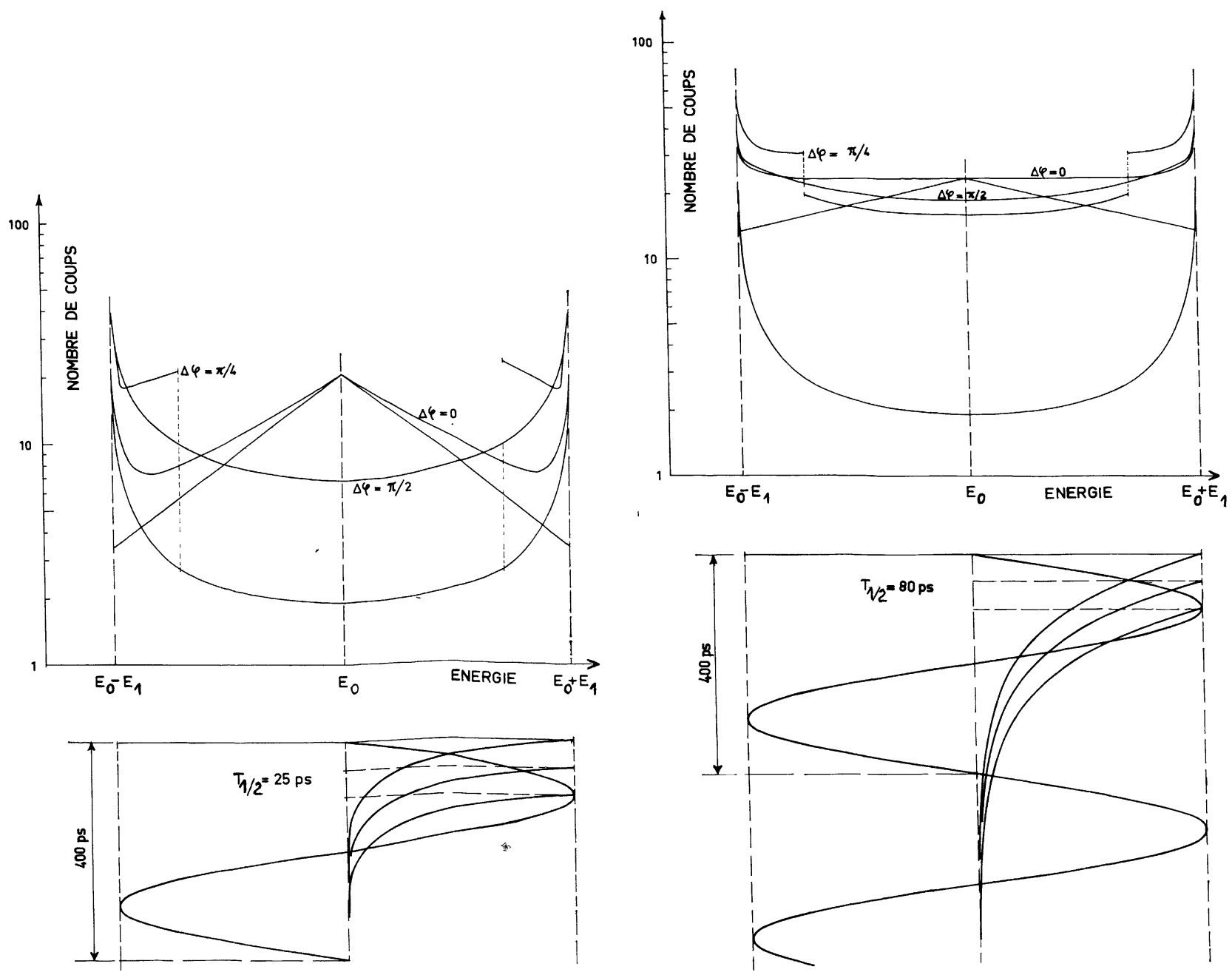

FIG. 3 et 4 . - Spectres de décroissance théorique pour trois différences de phase entre les champs modulateur et pulsateur $\left(T_{1 / 2}=25 \mathrm{ps}\right.$ et $\left.T_{1 / 2}=80 \mathrm{ps}\right)$.

phase, n'est pas exactement connue; ii) la résolution en énergie du détecteur (jonction $\mathrm{Si}-\mathrm{Li}$ ou spectromètre magnétique) par rapport au champ électrique maximal qu'il est possible de créer.

Dans la distribution spatiale du faisceau pulsé interviennent surtout la résolution en temps des paquets de particules conditionnée par la déflexion maximale, les qualités optiques du faisceau et le courant moyen sur la cible. La modification de la raie d'électrons $\mathrm{K}$ modulée, déclenchée par le faisceau pulsé, peut être représentée de façon simple dans le cas d'un détecteur de résolution nulle ( $f i g .3$ et 4 ).

Dans le cas de la double modulation, le calcul dépend d'un plus grand nombre de paramètres. Grâce à une méthode directe de simulation, il est possible de visua- liser la modification des spectres tridimensionnels, ce, dans différents cas : durée de vie très longue ou très courte vis-à-vis de la période du champ électrique ou au contraire comparable à celle-ci. Le procédé consiste à échantillonner simultanément deux sinusoïdes $50 \mathrm{~Hz}$ par deux convertisseurs multicanaux. On peut, en faisant varier pas à pas la différence de phase entre les sinusoïdes, construire la décroissance exponentielle avec une échelle de commande; en effet, la durée de vie et la période du champ n'interviennent que par leur rapport ( fig. 5).

L'avantage de la double modulation est de pouvoir réaliser une expérience multicanaux permettant d'utiliser le faisceau à $100 \%$ contre $20 \%$ dans le cas de la pulsation-modulation. 

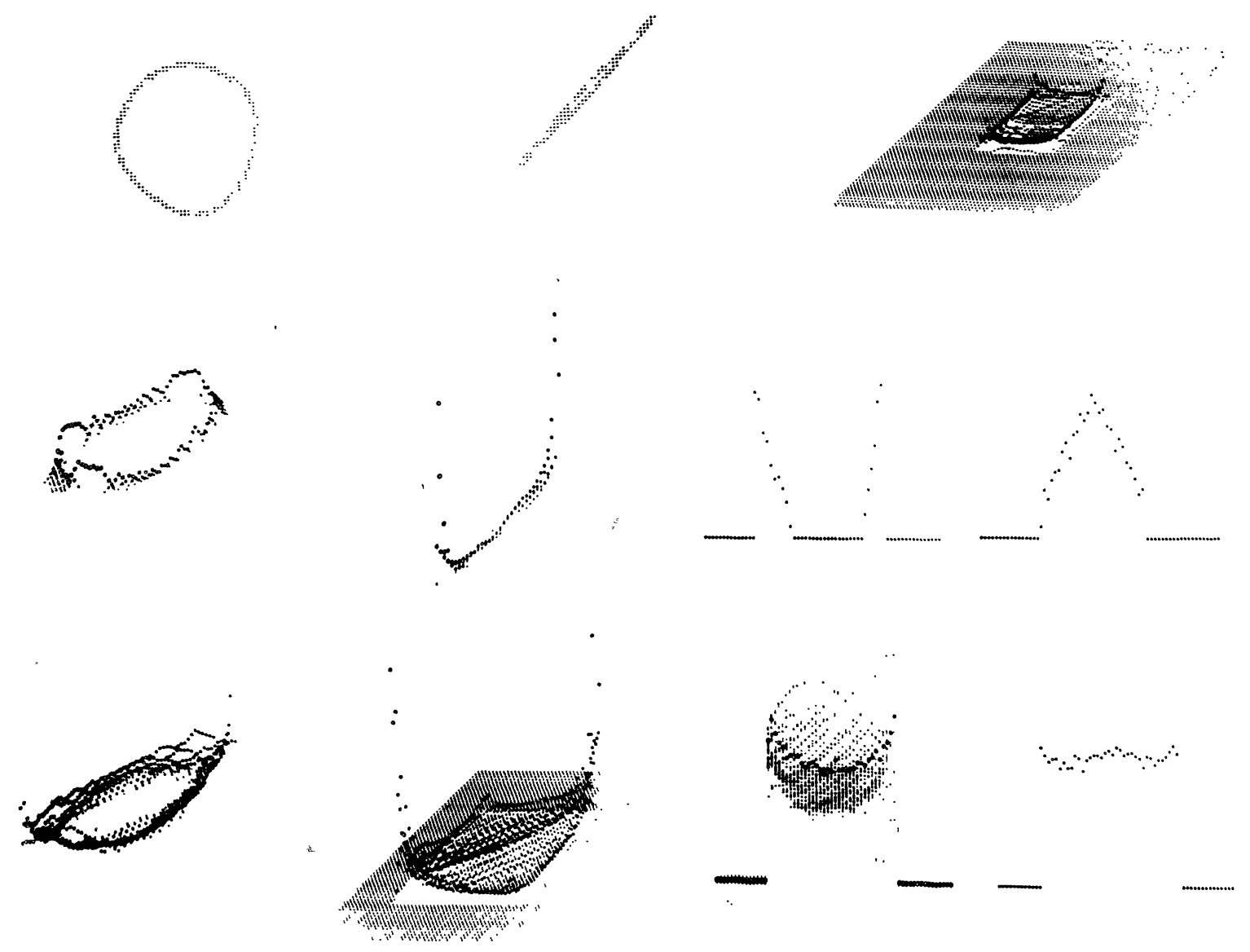

FIG. 5. - Spectres bidimensionnels (de gauche à droite et de haut en bas) :

a) "Prompte ", $\varphi=\pi / 2$; b) "prompte ", $\varphi=0$; c) $T_{1 / 2}$ infinie ; d) $T_{1 / 2}=8 \mathrm{ps}, \varphi=\pi / 2$; e) $T_{1 / 2}=8 \mathrm{ps}$, $\varphi=0$; f et $\mathrm{g}$ ) coupes médianes en échelle logarithmique des deux vues précédentes ; h) $T_{1 / 2}=40 \mathrm{ps}, \varphi=\pi / 2$; i) $T_{1 / 2}=40 \mathrm{ps}, \varphi=0 ; \mathrm{j}$ ) et $\mathrm{k}$ ) coupes médianes des deux vues précédentes.

\section{BIBLIOGRAPHIE}

[1] Blaugrund (A. E.) et al., Phys. Rev., 1960, 120, [3] Tové (P. A.) et Jonasson (L. G.), Coll. Électronique 1328. Nucléaire, 1963.

[2] Bein-Zvi (I.) et al., Nucl. Phys., 1968, A 117, 625. [4] GoRodeitzky (S.) et al., Nucl. Instr., $1967,57,152$. 